\title{
The Generation Model of Particle Physics
}

\author{
Brian Robson \\ Department of Theoretical Physics, Research School of Physics and Engineering, \\ The Australian National University, Canberra \\ Australia
}

\section{Introduction}

The main purpose of this chapter is to present an alternative to the Standard Model (SM) (Gottfried and Weisskopf, 1984) of particle physics. This alternative model, called the Generation Model (GM) (Robson, 2002; 2004; Evans and Robson, 2006), describes all the transition probabilities for interactions involving the six leptons and the six quarks, which form the elementary particles of the SM in terms of only three unified additive quantum numbers instead of the nine non-unified additive quantum numbers allotted to the leptons and quarks in the SM.

The chapter presents (Section 2) an outline of the current formulation of the SM: the elementary particles and the fundamental interactions of the SM, and the basic problem inherent in the SM. This is followed by (Section 3) a summary of the GM, highlighting the essential differences between the GM and the SM. Section 3 also introduces a more recent development of a composite GM in which both leptons and quarks have a substructure. This enhanced GM has been named the Composite Generation Model (CGM) (Robson, 2005; 2011a). In this chapter, for convenience, we shall refer to this enhanced GM as the CGM, whenever the substructure of leptons and quarks is important for the discussion. Section 4 focuses on several important consequences of the different paradigms provided by the GM. In particular: the origin of mass, the mass hierarchy of the leptons and quarks, the origin of gravity and the origin of apparent $\mathrm{CP}$ violation, are discussed. Finally, Section 5 provides a summary and discusses future prospects.

\section{Standard model of particle physics}

The Standard Model (SM) of particle physics (Gottfried and Weisskopf, 1984) was developed throughout the 20th century, although the current formulation was essentially finalized in the mid-1970s following the experimental confirmation of the existence of quarks (Bloom et al., 1969; Breidenbach et al., 1969).

The SM has enjoyed considerable success in describing the interactions of leptons and the multitude of hadrons (baryons and mesons) with each other as well as the decay modes of the unstable leptons and hadrons. However the model is considered to be incomplete in the sense that it provides no understanding of several empirical observations such as: the existence of three families or generations of leptons and quarks, which apart from mass have similar properties; the mass hierarchy of the elementary particles, which form the basis of the SM; the nature of the gravitational interaction and the origin of $\mathrm{CP}$ violation. 
In this section a summary of the current formulation of the SM is presented: the elementary particles and the fundamental interactions of the SM, and then the basic problem inherent in the SM.

\subsection{Elementary particles of the SM}

In the SM the elementary particles that are the constituents of matter are assumed to be the six leptons: electron neutrino $\left(v_{e}\right)$, electron $\left(e^{-}\right)$, muon neutrino $\left(v_{\mu}\right)$, muon $\left(\mu^{-}\right)$, tau neutrino $\left(v_{\tau}\right)$, tau $\left(\tau^{-}\right)$and the six quarks: up $(u)$, down $(d)$, charmed $(c)$, strange $(s)$, top $(t)$ and bottom $(b)$, together with their antiparticles. These twelve particles are all spin- $\frac{1}{2}$ particles and fall naturally into three families or generations: (i) $v_{e}, e^{-}, u, d$; (ii) $v_{\mu}, \mu^{-}, c, s$; (iii) $v_{\tau}, \tau^{-}, t, b$. Each generation consists of two leptons with charges $Q=0$ and $Q=-1$ and two quarks with charges $Q=+\frac{2}{3}$ and $Q=-\frac{1}{3}$. The masses of the particles increase significantly with each generation with the possible exception of the neutrinos, whose very small masses have yet to be determined.

In the SM the leptons and quarks are allotted several additive quantum numbers: charge $Q$, lepton number $L$, muon lepton number $L_{\mu}$, tau lepton number $L_{\tau}$, baryon number $A$, strangeness $S$, charm $C$, bottomness $B$ and topness $T$. These are given in Table 1 . For each particle additive quantum number $N$, the corresponding antiparticle has the additive quantum number $-N$.

\begin{tabular}{|lccccccccc|}
\hline particle & $Q$ & $L$ & $L_{\mu}$ & $L_{\tau}$ & $A$ & $S$ & $C$ & $B$ & $T$ \\
\hline$v_{e}$ & 0 & 1 & 0 & 0 & 0 & 0 & 0 & 0 & 0 \\
$e^{-}$ & -1 & 1 & 0 & 0 & 0 & 0 & 0 & 0 & 0 \\
$v_{\mu}$ & 0 & 1 & 1 & 0 & 0 & 0 & 0 & 0 & 0 \\
$\mu^{-}$ & -1 & 1 & 1 & 0 & 0 & 0 & 0 & 0 & 0 \\
$v_{\tau}$ & 0 & 1 & 0 & 1 & 0 & 0 & 0 & 0 & 0 \\
$\tau^{-}$ & -1 & 1 & 0 & 1 & 0 & 0 & 0 & 0 & 0 \\
$u$ & $+\frac{2}{3}$ & 0 & 0 & 0 & $\frac{1}{3}$ & 0 & 0 & 0 & 0 \\
$d$ & $-\frac{1}{3}$ & 0 & 0 & 0 & $\frac{1}{3}$ & 0 & 0 & 0 & 0 \\
$c$ & $+\frac{2}{3}$ & 0 & 0 & 0 & $\frac{1}{3}$ & 0 & 1 & 0 & 0 \\
$s$ & $-\frac{1}{3}$ & 0 & 0 & 0 & $\frac{1}{3}$ & -1 & 0 & 0 & 0 \\
$t$ & $+\frac{2}{3}$ & 0 & 0 & 0 & $\frac{1}{3}$ & 0 & 0 & 0 & 1 \\
$b$ & $-\frac{1}{3}$ & 0 & 0 & 0 & $\frac{1}{3}$ & 0 & 0 & -1 & 0 \\
\hline
\end{tabular}

Table 1. SM additive quantum numbers for leptons and quarks

Table 1 demonstrates that, except for charge, leptons and quarks are allotted different kinds of additive quantum numbers so that this classification of the elementary particles in the SM is non-unified.

The additive quantum numbers $Q$ and $A$ are assumed to be conserved in strong, electromagnetic and weak interactions. The lepton numbers $L, L_{\mu}$ and $L_{\tau}$ are not involved in strong interactions but are strictly conserved in both electromagnetic and weak interactions. The remainder, $S, C, B$ and $T$ are strictly conserved only in strong and electromagnetic interactions but can undergo a change of one unit in weak interactions.

The quarks have an additional additive quantum number called "color charge", which can take three values so that in effect we have three kinds of each quark, $u, d$, etc. These are often 
called red, green and blue quarks. The antiquarks carry anticolors, which for simplicity are called antired, antigreen and antiblue. Each quark or antiquark carries a single unit of color or anticolor charge, respectively. The leptons do not carry a color charge and consequently do not participate in the strong interactions, which occur between particles carrying color charges.

\subsection{Fundamental interactions of the SM}

The SM recognizes four fundamental interactions in nature: strong, electromagnetic, weak and gravity. Since gravity plays no role in particle physics because it is so much weaker than the other three fundamental interactions, the SM does not attempt to explain gravity. In the SM the other three fundamental interactions are assumed to be associated with a local gauge field.

\subsubsection{Strong interactions}

The strong interactions, mediated by massless neutral spin-1 gluons between quarks carrying a color charge, are described by an SU(3) local gauge theory called quantum chromodynamics (QCD) (Halzen and Martin, 1984). There are eight independent kinds of gluons, each of which carries a combination of a color charge and an anticolor charge (e.g. red-antigreen). The strong interactions between color charges are such that in nature the quarks (antiquarks) are grouped into composites of either three quarks (antiquarks), called baryons (antibaryons), each having a different color (anticolor) charge or a quark-antiquark pair, called mesons, of opposite color charges. In the $S U(3)$ color gauge theory each baryon, antibaryon or meson is colorless. However, these colorless particles, called hadrons, may interact strongly via residual strong interactions arising from their composition of colored quarks and/or antiquarks. On the other hand the colorless leptons are assumed to be structureless in the SM and consequently do not participate in strong interactions.

\subsubsection{Electromagnetic interactions}

The electromagnetic interactions, mediated by massless neutral spin-1 photons between electrically charged particles, are described by a $U(1)$ local gauge theory called quantum electrodynamics (Halzen and Martin, 1984).

\subsubsection{Weak interactions}

The weak interactions, mediated by the massive $W^{+}, W^{-}$and $Z^{0}$ vector bosons between all the elementary particles of the SM, fall into two classes: (i) charge-changing (CC) weak interactions involving the $W^{+}$and $W^{-}$bosons and (ii) neutral weak interactions involving the $Z^{0}$ boson. The CC weak interactions, acting exclusively on left-handed particles and right-handed antiparticles, are described by an $S U(2)_{L}$ local gauge theory, where the subscript $L$ refers to left-handed particles only (Halzen and Martin, 1984). On the other hand, the neutral weak interactions act on both left-handed and right-handed particles, similar to the electromagnetic interactions. In fact the SM assumes (Glashow, 1961) that both the $Z^{0}$ and the photon $(\gamma)$ arise from a mixing of two bosons, $W^{0}$ and $B^{0}$, via an electroweak mixing angle $\theta_{W}$ :

$$
\begin{gathered}
\gamma=B^{0} \cos \theta_{W}+W^{0} \sin \theta_{W} \\
Z^{0}=-B^{0} \sin \theta_{W}+W^{0} \cos \theta_{W} .
\end{gathered}
$$


These are described by a $U(1) \times S U(2)_{L}$ local gauge theory, where the $U(1)$ symmetry involves both left-handed and right-handed particles.

Experiment requires the masses of the weak gauge bosons, $W$ and $Z$, to be heavy so that the weak interactions are very short-ranged. On the other hand, Glashow's proposal, based upon the concept of a non-Abelian SU(2) Yang-Mills gauge theory, requires the mediators of the weak interactions to be massless like the photon. This boson mass problem was resolved by Weinberg (1967) and Salam (1968), who independently employed the idea of spontaneous symmetry breaking involving the Higgs mechanism (Englert and Brout, 1964; Higgs, 1964). In this way the $W$ and $Z$ bosons acquire mass and the photon remains massless.

The above treatment of the electromagnetic and weak interactions in terms of a $U(1) \times S U(2)_{L}$ local gauge theory has become known as the Glashow, Weinberg and Salam (GWS) model and forms one of the cornerstones of the SM. The model gives the relative masses of the $W$ and $Z$ bosons in terms of the electroweak mixing angle:

$$
M_{W}=M_{Z} \cos \theta_{W} .
$$

The Higgs mechanism was also able to cure the associated fermion mass problem (Aitchison and Hey, 1982): the finite masses of the leptons and quarks cause the Lagrangian describing the system to violate the $S U(2)_{L}$ gauge invariance. By coupling originally massless fermions to a scalar Higgs field, it is possible to produce the observed physical fermion masses without violating the gauge invariance. However, the GWS model requires the existence of a new massive spin zero boson, the Higgs boson, which to date remains to be detected. In addition, the fermion-Higgs coupling strength is dependent upon the mass of the fermion so that a new parameter is required for each fermion mass in the theory.

In 1971, t'Hooft (1971a,b) showed that the GWS model of the electroweak interactions was renormalizable and this self-consistency of the theory led to its general acceptance. In 1973, events corresponding to the predicted neutral currents mediated by the $Z^{0}$ boson were observed (Hasert et al., 1973; 1974), while bosons, with approximately the expected masses, were discovered in 1983 (Arnison et al., 1983; Banner et al., 1983), thereby confirming the GWS model.

Another important property of the CC weak interactions is their universality for both leptonic and hadronic processes. In the SM this property is taken into account differently for leptonic and hadronic processes.

For leptonic CC weak interaction processes, each of the charged leptons is assumed to form a weak isospin doublet $\left(i=\frac{1}{2}\right)$ with its respective neutrino, i.e. $\left(v_{e}, e^{-}\right),\left(v_{\mu}, \mu^{-}\right),\left(v_{\tau}, \tau^{-}\right)$, with each doublet having the third component of weak isospin $i_{3}=\left(+\frac{1}{2},-\frac{1}{2}\right)$. In addition each doublet is associated with a different lepton number so that there are no CC weak interaction transitions between generations. Thus for leptonic processes, the concept of a universal CC weak interaction allows one to write (for simplicity we restrict the discussion to the first two generations only):

$$
a\left(v_{e}, e^{-} ; W^{-}\right)=a\left(v_{\mu}, \mu^{-} ; W^{-}\right)=g_{w} .
$$

Here $a\left(\alpha, \beta ; W^{-}\right)$represents the CC weak interaction transition amplitude involving the fermions $\alpha, \beta$ and the $W^{-}$boson, and $g_{w}$ is the universal CC weak interaction transition 
amplitude. Lepton number conservation gives

$$
a\left(v_{e}, \mu^{-} ; W^{-}\right)=a\left(v_{\mu}, e^{-} ; W^{-}\right)=0,
$$

so that there are no CC weak interaction transitions between generations in agreement with experiment.

Unlike the pure leptonic decays, which are determined by the conservation of the various lepton numbers, there is no quantum number in the SM which restricts quark (hadronic) CC weak interaction processes between generations. In the SM the quarks do not appear to form weak isospin doublets: the known decay processes of neutron $\beta$-decay and $\Lambda^{0} \beta$-decay suggest that quarks mix between generations and that the "flavor" quantum numbers, $S, C, B$ and $T$ are not necessarily conserved in CC weak interaction processes.

In the SM neutron $\beta$-decay:

$$
n^{0} \rightarrow p^{+}+e^{-}+\bar{v}_{e}
$$

is interpreted as the sequential transition

$$
d \rightarrow u+W^{-}, \quad W^{-} \rightarrow e^{-}+\bar{v}_{e} .
$$

The overall coupling strength of the CC weak interactions involved in neutron $\beta$-decay was found to be slightly weaker $(\approx 0.95)$ than that for muon decay:

$$
\mu^{-} \rightarrow v_{\mu}+W^{-}, \quad W^{-} \rightarrow e^{-}+\bar{v}_{e} .
$$

Similarly, $\Lambda^{0} \beta$-decay:

$$
\Lambda^{0} \rightarrow p^{+}+e^{-}+\bar{v}_{e},
$$

is interpreted in the SM as the sequential transition

$$
s \rightarrow u+W^{-}, \quad W^{-} \rightarrow e^{-}+\bar{v}_{e} .
$$

In this case the overall coupling strength of the CC weak interactions was found to be significantly less $(\approx 0.05)$ than that for muon decay.

In the SM the universality of the CC weak interaction for both leptonic and hadronic processes is restored by adopting the proposal of Cabibbo (1963) that in hadronic processes the CC weak interaction is shared between $\Delta S=0$ and $\Delta S=1$ transition amplitudes in the ratio of $\cos \theta_{c}$ : $\sin \theta_{c}$. The Cabibbo angle $\theta_{c}$ has a value $\approx 13^{0}$, which gives good agreement with experiment for the decay processes (7) and (10) relative to (8).

This "Cabibbo mixing" is an integral part of the SM. In the quark model it leads to a sharing of the CC weak interaction between quarks with different flavors (different generations) unlike the corresponding case of leptonic processes. Again, in order to simplify matters, the following discussion (and also throughout the chapter) will be restricted to the first two generations of the elementary particles of the SM, involving only the Cabibbo mixing, although the extension to three generations is straightforward (Kobayashi and Maskawa, 1973). In the latter case, the quark mixing parameters correspond to the so-called Cabibbo-Kobayashi-Maskawa (CKM) matrix elements, which indicate that inclusion of the 
third generation would have a minimal effect on the overall coupling strength of the CC weak interactions.

Cabibbo mixing was incorporated into the quark model of hadrons by postulating that the so-called weak interaction eigenstate quarks, $d^{\prime}$ and $s^{\prime}$, form CC weak interaction isospin doublets with the $u$ and $c$ quarks, respectively: $\left(u, d^{\prime}\right)$ and $\left(c, s^{\prime}\right)$. These weak eigenstate quarks are linear superpositions of the so-called mass eigenstate quarks $(d$ and $s)$ :

$$
d^{\prime}=d \cos \theta_{c}+s \sin \theta_{c}
$$

and

$$
s^{\prime}=-d \sin \theta_{c}+s \cos \theta_{c} .
$$

The quarks $d$ and $s$ are the quarks which participate in the electromagnetic and the strong interactions with the full allotted strengths of electric charge and color charge, respectively. The quarks $d^{\prime}$ and $s^{\prime}$ are the quarks which interact with the $u$ and $c$ quarks, respectively, with the full strength of the CC weak interaction.

In terms of transition amplitudes, Eqs. (11) and (12) can be represented as

$$
a\left(u, d^{\prime} ; W^{-}\right)=a\left(u, d ; W^{-}\right) \cos \theta_{c}+a\left(u, s ; W^{-}\right) \sin \theta_{\mathcal{c}}=g_{w}
$$

and

$$
a\left(c, s^{\prime} ; W^{-}\right)=-a\left(c, d ; W^{-}\right) \sin \theta_{c}+a\left(c, s ; W^{-}\right) \cos \theta_{c}=g_{w} .
$$

In addition one has the relations

$$
a\left(u, s^{\prime} ; W^{-}\right)=-a\left(u, d ; W^{-}\right) \sin \theta_{c}+a\left(u, s ; W^{-}\right) \cos \theta_{c}=0
$$

and

$$
a\left(c, d^{\prime} ; W^{-}\right)=a\left(c, d ; W^{-}\right) \cos \theta_{c}+a\left(c, s ; W^{-}\right) \sin \theta_{c}=0 .
$$

Eqs. (13) and (14) indicate that it is the $d^{\prime}$ and $s^{\prime}$ quarks which interact with the $u$ and $c$ quarks, respectively, with the full strength $g_{w}$. These equations for quarks correspond to Eq. (4) for leptons. Similarly, Eqs. (15) and (16) for quarks correspond to Eq. (5) for leptons. However, there is a fundamental difference between Eqs. (15) and (16) for quarks and Eq. (5) for leptons. The former equations do not yield zero amplitudes because there exists some quantum number (analagous to muon lepton number) which is required to be conserved. This lack of a selection rule indicates that the notion of weak isospin symmetry for the doublets $\left(u, d^{\prime}\right)$ and $\left(c, s^{\prime}\right)$ is dubious.

Eqs. (13) and (15) give

$$
a\left(u, d ; W^{-}\right)=g_{w} \cos \theta_{c}, \quad a\left(u, s ; W^{-}\right)=g_{w} \sin \theta_{c} .
$$

Thus in the two generation approximation of the SM, transitions involving $d \rightarrow u+W^{-}$ proceed with a strength proportional to $g_{w}^{2} \cos ^{2} \theta_{c} \approx 0.95 g_{w}^{2}$, while transitions involving $s \rightarrow u+W^{-}$proceed with a strength proportional to $g_{w}^{2} \sin ^{2} \theta_{c} \approx 0.05 g_{w}^{2}$, as required by experiment. 


\subsection{Basic problem inherent in SM}

The basic problem with the SM is the classification of its elementary particles employing a diverse complicated scheme of additive quantum numbers (Table 1), some of which are not conserved in weak interaction processes; and at the same time failing to provide any physical basis for this scheme.

A good analogy of the SM situation is the Ptolemaic model of the universe, based upon a stationary Earth at the center surrounded by a rotating system of crystal spheres refined by the addition of epicycles (small circular orbits) to describe the peculiar movements of the planets around the Earth. While the Ptolemaic model yielded an excellent description, it is a complicated diverse scheme for predicting the movements of the Sun, Moon, planets and the stars around a stationary Earth and unfortunately provides no understanding of these complicated movements.

Progress in understanding the universe was only made when the Ptolemaic model was replaced by the Copernican-Keplerian model, in which the Earth moved like the other planets around the Sun, and Newton discovered his universal law of gravitation to describe the approximately elliptical planetary orbits.

The next section describes a new model of particle physics, the Generation Model (GM), which addresses the problem within the SM, replacing it with a much simpler and unified classification scheme of leptons and quarks, and providing some understanding of phenomena, which the SM is unable to address.

\section{Generation model of particle physics}

The Generation Model (GM) of particle physics has been developed over the last decade. In the initial paper (Robson, 2002) a new classification of the elementary particles, the six leptons and the six quarks, of the SM was proposed. This classification was based upon the use of only three additive quantum numbers: charge $(Q)$, particle number $(p)$ and generation quantum number $(g)$, rather than the nine additive quantum numbers (see Table 1 ) of the SM. Thus the new classification is both simpler and unified in that leptons and quarks are assigned the same kind of additive quantum numbers unlike those of the SM. It will be discussed in more detail in Subsection 3.1.

Another feature of the new classification scheme is that all three additive quantum numbers, $Q, p$ and $g$, are required to be conserved in all leptonic and hadronic processes. In particular the generation quantum number $g$ is strictly conserved in weak interactions unlike some of the quantum numbers, e.g. strangeness $S$, of the SM. This latter requirement led to a new treatment of quark mixing in hadronic processes (Robson, 2002; Evans and Robson, 2006), which will be discussed in Subsection 3.2.

The development of the GM classification scheme, which provides a unified description of leptons and quarks, indicated that leptons and quarks are intimately related and led to the development of composite versions of the GM, which we refer to as the Composite Generation Model (CGM) (Robson, 2005; 2011a). The CGM will be discussed in Subsection 3.3.

Subsection 3.4 discusses the fundamental interactions of the GM. 


\subsection{Unified classification of leptons and quarks}

Table 2 displays a set of three additive quantum numbers: charge $(Q)$, particle number $(p)$ and generation quantum number $(g)$ for the unified classification of the leptons and quarks corresponding to the current CGM (Robson, 2011a). As for Table 1 the corresponding antiparticles have the opposite sign for each particle additive quantum number.

\begin{tabular}{|lccc|cccc|}
\hline particle & $Q$ & $p$ & $g$ & particle & $Q$ & $p$ & $g$ \\
\hline$v_{e}$ & 0 & -1 & 0 & $u$ & $+\frac{2}{3}$ & $\frac{1}{3}$ & 0 \\
$e^{-}$ & -1 & -1 & 0 & $d$ & $-\frac{1}{3}$ & $\frac{1}{3}$ & 0 \\
\hline$v_{\mu}$ & 0 & -1 & \pm 1 & $c$ & $+\frac{2}{3}$ & $\frac{1}{3}$ & \pm 1 \\
$\mu^{-}$ & -1 & -1 & \pm 1 & $s$ & $-\frac{1}{3}$ & $\frac{1}{3}$ & \pm 1 \\
\hline$v_{\tau}$ & 0 & -1 & $0, \pm 2$ & $t$ & $+\frac{2}{3}$ & $\frac{1}{3}$ & $0, \pm 2$ \\
$\tau^{-}$ & -1 & -1 & $0, \pm 2$ & $b$ & $-\frac{1}{3}$ & $\frac{1}{3}$ & $0, \pm 2$ \\
\hline
\end{tabular}

Table 2. CGM additive quantum numbers for leptons and quarks

Each generation of leptons and quarks has the same set of values for the additive quantum numbers $Q$ and $p$. The generations are differentiated by the generation quantum number $g$, which in general can have multiple values. The latter possibilities arise from the composite nature of the leptons and quarks in the CGM.

The three conserved additive quantum numbers, $Q, p$ and $g$ are sufficient to describe all the observed transition amplitudes for both hadronic and leptonic processes, provided each "force" particle, mediating the various interactions, has $p=g=0$.

Comparison of Tables 1 and 2 indicates that the two models, SM and CGM, have only one additive quantum number in common, namely electric charge $Q$, which serves the same role in both models and is conserved. The second additive quantum number of the CGM, particle number $p$, replaces both lepton number $L$ and baryon number $A$ of the SM. The third additive quantum number of the CGM, generation quantum number $g$, effectively replaces the remaining additive quantum numbers of the $S M, L_{\mu}, L_{\tau}, S, C, B$ and $T$.

Table 2 shows that the CGM provides both a simpler and unified classification scheme for leptons and quarks. Furthermore, the generation quantum number $g$ is conserved in the CGM unlike the additive quantum numbers, $S, C, B$ and $T$ of the SM. Conservation of $g$ requires a new treatment of quark mixing in hadronic processes, which will be discussed in the next subsection.

\subsection{Quark mixing in hadronic CC weak interaction processes in the GM}

The GM differs from the SM in two fundamental ways, which are essential to preserve the universality of the CC weak interaction for both leptonic and hadronic processes. In the SM this was accomplished, initially by Cabibbo (1963) for the first two generations by the introduction of "Cabibbo quark mixing", and later by Kobayashi and Maskawa (1973), who generalized quark mixing involving the CKM matrix elements to the three generations.

Firstly, the GM postulates that the mass eigenstate quarks of the same generation, e.g. $(u, d)$, form weak isospin doublets and couple with the full strength of the CC weak interaction, $g_{w}$, like the lepton doublets, e.g. $\left(v_{e}, e^{-}\right)$. Unlike the SM, the GM requires that there is no 
coupling between mass eigenstate quarks from different generations. This latter requirement corresponds to the conservation of the generation quantum number $g$ in the CC weak interaction processes.

Secondly, the GM postulates that hadrons are composed of weak eigenstate quarks such as $d^{\prime}$ and $s^{\prime}$ given by Eqs. (11) and (12) in the two generation approximation, rather than the corresponding mass eigenstate quarks, $d$ and $s$, as in the SM.

To maintain lepton-quark universality for CC weak interaction processes in the two generation approximation, the GM postulates that

$$
a\left(u, d ; W^{-}\right)=a\left(c, s ; W^{-}\right)=g_{w}
$$

and generation quantum number conservation gives

$$
a\left(u, s ; W^{-}\right)=a\left(c, d ; W^{-}\right)=0 .
$$

Eqs. (18) and (19) are the analogues of Eqs. (4) and (5) for leptons. Thus the quark pairs $(u, d)$ and $(c, s)$ in the GM form weak isospin doublets, similar to the lepton pairs $\left(v_{e}, e^{-}\right)$and $\left(v_{\mu}, \mu^{-}\right)$, thereby establishing a close lepton-quark parallelism with respect to weak isospin symmetry.

To account for the reduced transition probabilities for neutron and $\Lambda^{0} \beta$-decays, the GM postulates that the neutron and $\Lambda^{0}$ baryon are composed of weak eigenstate quarks, $u, d^{\prime}$ and $s^{\prime}$. Thus, neutron $\beta$-decay is to be interpreted as the sequential transition

$$
d^{\prime} \rightarrow u+W^{-}, \quad W^{-} \rightarrow e^{-}+\bar{v}_{e} .
$$

The primary transition has the amplitude $a\left(u, d^{\prime} ; W^{-}\right)$given by

$$
a\left(u, d^{\prime} ; W^{-}\right)=a\left(u, d ; W^{-}\right) \cos \theta_{c}+a\left(u, s ; W^{-}\right) \sin \theta_{c}=g_{w} \cos \theta_{c},
$$

where we have used Eqs. (18) and (19). This gives the same transition probability for neutron $\beta$-decay $\left(g_{w}^{4} \cos ^{2} \theta_{c}\right)$ relative to muon decay $\left(g_{w}^{4}\right)$ as the SM. Similarly, $\Lambda^{0} \beta$-decay is to be interpreted as the sequential transition

$$
s^{\prime} \rightarrow u+W^{-}, \quad W^{-} \rightarrow e^{-}+\bar{v}_{e} .
$$

In this case the primary transition has the amplitude $a\left(u, s^{\prime} ; W^{-}\right)$given by

$$
a\left(u, s^{\prime} ; W^{-}\right)=-a\left(u, d ; W^{-}\right) \sin \theta_{c}+a\left(u, s ; W^{-}\right) \cos \theta_{c}=-g_{w} \sin \theta_{c} .
$$

Thus $\Lambda^{0} \beta$-decay has the same transition probability $\left(g_{w}^{4} \sin ^{2} \theta_{c}\right)$ relative to muon decay $\left(g_{w}^{4}\right)$ as that given by the SM.

The GM differs from the SM in that it treats quark mixing differently from the method introduced by Cabibbo (1963) and employed in the SM. Essentially, in the GM, the quark mixing is placed in the quark states (wave functions) rather than in the CC weak interactions. This allows a unified and simpler classification of both leptons and quarks in terms of only three additive quantum numbers, $Q, p$ and $g$, each of which is conserved in all interactions. 


\subsection{Composite generation model}

The unified classification scheme of the GM makes feasible a composite version of the GM (CGM) (Robson, 2005). This is not possible in terms of the non-unified classification scheme of the SM, involving different additive quantum numbers for leptons than for quarks and the non-conservation of some additive quantum numbers, such as strangeness, in the case of quarks. Here we shall present the current version (Robson, 2011a), which takes into account the mass hierarchy of the three generations of leptons and quarks. There is evidence that leptons and quarks, which constitute the elementary particles of the SM, are actually composites.

Firstly, the electric charges of the electron and proton are opposite in sign but are exactly equal in magnitude so that atoms with the same number of electrons and protons are neutral. Consequently, in a proton consisting of quarks, the electric charges of the quarks are intimately related to that of the electron: in fact, the up quark has charge $Q=+\frac{2}{3}$ and the down quark has charge $Q=-\frac{1}{3}$, if the electron has electric charge $Q=-1$. These relations are readily comprehensible if leptons and quarks are composed of the same kinds of particles.

Secondly, the leptons and quarks may be grouped into three generations: (i) $\left(v_{e}, e^{-}, u, d\right)$, (ii) $\left(v_{\mu}, \mu^{-}, c, s\right)$ and (iii) $\left(v_{\tau}, \tau^{-}, t, b\right)$, with each generation containing particles which have similar properties. Corresponding to the electron, $e^{-}$, the second and third generations include the muon, $\mu^{-}$, and the tau particle, $\tau^{-}$, respectively. Each generation contains a neutrino associated with the corresponding leptons: the electron neutrino, $v_{e}$, the muon neutrino, $v_{\mu}$, and the tau neutrino, $v_{\tau}$. In addition, each generation contains a quark with $Q=+\frac{2}{3}$ (the $u$, $c$ and $t$ quarks) and a quark with $Q=-\frac{1}{3}$ (the $d, s$ and $b$ quarks). Each pair of leptons, e.g. $\left(v_{e}, e^{-}\right)$, and each pair of quarks, e.g. $(u, d)$, are connected by isospin symmetries, otherwise the grouping into the three families is according to increasing mass of the corresponding family members. The existence of three repeating patterns suggests strongly that the members of each generation are composites.

Thirdly, the GM, which provides a unified classification scheme for leptons and quarks, also indicates that these particles are intimately related. It has been demonstrated (Robson, 2004) that this unified classification scheme leads to a relation between strong isospin $(I)$ and weak isospin (i) symmetries. In particular, their third components are related by an equation:

$$
i_{3}=I_{3}+\frac{1}{2} g,
$$

where $g$ is the generation quantum number. In addition, electric charge is related to $I_{3}, p, g$ and $i_{3}$ by the equations:

$$
Q=I_{3}+\frac{1}{2}(p+g)=i_{3}+\frac{1}{2} p .
$$

These relations are valid for both leptons and quarks and suggest that there exists an underlying flavor $S U(3)$ symmetry. The simplest conjecture is that this new flavor symmetry is connected with the substructure of leptons and quarks, analogous to the flavor $S U(3)$ symmetry underlying the quark structure of the lower mass hadrons in the Eightfold Way (Gell-Mann and Ne'eman, 1964).

The CGM description of the first generation is based upon the two-particle models of Harari (1979) and Shupe (1979), which are very similar and provide an economical and impressive 
description of the first generation of leptons and quarks. Both models treat leptons and quarks as composites of two kinds of spin- $1 / 2$ particles, which Harari named "rishons" from the Hebrew word for first or primary. This name has been adopted for the constituents of leptons and quarks. The CGM is constructed within the framework of the GM, i.e. the same kind of additive quantum numbers are assigned to the constituents of both leptons and quarks, as were previously allotted in the GM to leptons and quarks (see Table 2).

In the Harari-Shupe Model (HSM), two elementary spin-1/2 rishons and their corresponding antiparticles are employed to construct the leptons and quarks: (i) a $T$-rishon with $Q=+1 / 3$ and (ii) a $V$-rishon with $Q=0$. Their antiparticles (denoted in the usual way by a bar over the defining particle symbol) are a $\bar{T}$-antirishon with $Q=-1 / 3$ and a $\bar{V}$-antirishon with $Q=0$, respectively. Each spin-1/2 lepton and quark is composed of three rishons/antirishons.

Table 3 shows the proposed structures of the first generation of leptons and quarks in the HSM.

\begin{tabular}{|ccc|}
\hline particle & structure & $Q$ \\
\hline$e^{+}$ & $T T T$ & +1 \\
$u$ & $T T V, T V T, V T T$ & $+\frac{2}{3}$ \\
$\bar{d}$ & $T V V, V T V, V V T$ & $+\frac{1}{3}$ \\
$v_{e}$ & $V V V$ & 0 \\
$\overline{v_{e}}$ & $\bar{V} \bar{V} \bar{V}$ & 0 \\
$d$ & $\bar{T} \bar{V} \bar{V}, \bar{V} \bar{T} \bar{V}, \bar{V} \bar{V} \bar{T}$ & $-\frac{1}{3}$ \\
$\bar{u}$ & $\bar{T} \bar{T} \bar{V}, \bar{T} \bar{V} \bar{T}, \bar{V} \bar{T} \bar{T}$ & $-\frac{2}{3}$ \\
$e^{-}$ & $\bar{T} \bar{T} \bar{T}$ & -1 \\
\hline
\end{tabular}

Table 3. HSM of first generation of leptons and quarks

It should be noted that no composite particle involves mixtures of rishons and antirishons, as emphasized by Shupe. Both Harari and Shupe noted that quarks contained mixtures of the two kinds of rishons, whereas leptons did not. They concluded that the concept of color related to the different internal arrangements of the rishons in a quark: initially the ordering TTV, TVT and VTT was associated with the three colors of the $u$-quark. However, at this stage, no underlying mechanism was suggested for color. Later, a dynamical basis was proposed by Harari and Seiberg (1981), who were led to consider color-type local gauged $S U(3)$ symmetries, namely $S U(3)_{C} \times S U(3)_{H}$, at the rishon level. They proposed a new super-strong color-type (hypercolor) interaction corresponding to the $S U(3)_{H}$ symmetry, mediated by massless hypergluons, which is responsible for binding rishons together to form hypercolorless leptons or quarks. This interaction was assumed to be analogous to the strong color interaction of the SM, mediated by massless gluons, which is responsible for binding quarks together to form baryons or mesons. However, in this dynamical rishon model, the color force corresponding to the $S U(3)_{C}$ symmetry is also retained, with the T-rishons and $V$-rishons carrying colors and anticolors. respectively, so that leptons are colorless but quarks are colored. Similar proposals were made by others (Casalbuoni and Gatto, 1980; Squires, $1980 ; 1981)$. In each of these proposals, both the color force and the new hypercolor interaction are assumed to exist independently of one another so that the original rishon model loses some of its economical description. Furthermore, the HSM does not provide a satisfactory understanding of the second and third generations of leptons and quarks. 


\begin{tabular}{|cccc|}
\hline rishon & $Q$ & $p$ & $g$ \\
\hline$T$ & $+\frac{1}{3}$ & $+\frac{1}{3}$ & 0 \\
$V$ & 0 & $+\frac{1}{3}$ & 0 \\
$U$ & 0 & $+\frac{1}{3}$ & -1 \\
\hline
\end{tabular}

Table 4. CGM additive quantum numbers for rishons

In order to overcome some of the deficiencies of the simple HSM, the two-rishon model was extended (Robson, 2005; 2011a), within the framework of the GM, in several ways.

Firstly, following the suggested existence of an $S U(3)$ flavor symmetry underlying the substructure of leptons and quarks by Eq. (25), a third type of rishon, the $U$-rishon, is introduced. This $U$-rishon has $Q=0$ but carries a non-zero generation quantum number, $g=-1$ (both the $T$-rishon and the $V$-rishon are assumed to have $g=0$ ). Thus, the CGM treats leptons and quarks as composites of three kinds of spin- $1 / 2$ rishons, although the $U$-rishon is only involved in the second and third generations.

Secondly, in the CGM, each rishon is allotted both a particle number $p$ and a generation quantum number $g$. Table 4 gives the three additive quantum numbers allotted to the three kinds of rishons. It should be noted that for each rishon additive quantum number $N$, the corresponding antirishon has the additive quantum number $-N$.

Historically, the term "particle" defines matter that is naturally occurring, especially electrons. In the CGM it is convenient to define a matter "particle" to have $p>0$, with the antiparticle having $p<0$. This definition of a matter particle leads to a modification of the HSM structures of the leptons and quarks which comprise the first generation. Essentially, the roles of the $V$-rishon and its antiparticle $\bar{V}$ are interchanged in the CGM compared with the HSM. Table 5 gives the CGM structures for the first generation of leptons and quarks. The particle number $p$ is clearly given by $\frac{1}{3}$ (number of rishons - number of antirishons). Thus the $u$-quark has $p=+\frac{1}{3}$, since it contains two $T$-rishons and one $\bar{V}$-antirishon. It should be noted that it is essential for the $u$-quark to contain a $\bar{V}$-antirishon $\left(p=-\frac{1}{3}\right)$ rather than a $V$-rishon $\left(p=+\frac{1}{3}\right)$ to obtain a value of $p=+\frac{1}{3}$, corresponding to baryon number $A=+\frac{1}{3}$ in the SM.

In the CGM, no significance is attached to the ordering of the $T$-rishons and the $\bar{V}$-antirishons (compare HSM) so that, e.g. the structures $T T \bar{V}, T \bar{V} T$ and $\bar{V} T T$ for the $u$-quark are considered to be equivalent. The concept of color is treated differently in the CGM: it is assumed that all three rishons, $T, V$ and $U$ carry a color charge, red, green or blue, while their antiparticles carry an anticolor charge, antired, antigreen or antiblue. The CGM postulates a strong color-type interaction corresponding to a local gauged $S U(3)_{C}$ symmetry (analogous to QCD) and mediated by massless hypergluons, which is responsible for binding rishons and antirishons together to form colorless leptons and colored quarks. The proposed structures of the quarks requires the composite quarks to have a color charge so that the dominant residual interaction between quarks is essentially the same as that between rishons, and consequently the composite quarks behave very like the elementary quarks of the SM. In the CGM we retain the term "hypergluon" as the mediator of the strong color interaction, rather than the term "gluon" employed in the SM, because it is the rishons rather than the quarks, which carry an elementary color charge.

In the CGM each lepton of the first generation (Table 5) is assumed to be colorless, consisting of three rishons (or antirishons), each with a different color (or anticolor), analogous to the 


\begin{tabular}{|lcccc|}
\hline particle & structure & $Q$ & $p$ & $g$ \\
\hline$e^{+}$ & $T T T$ & +1 & +1 & 0 \\
$u$ & $T T \bar{V}$ & $+\frac{2}{3}$ & $+\frac{1}{3}$ & 0 \\
$\bar{d}$ & $T \bar{V} \bar{V}$ & $+\frac{1}{3}$ & $-\frac{1}{3}$ & 0 \\
$v_{e}$ & $\bar{V} \bar{V} \bar{V}$ & 0 & -1 & 0 \\
$\overline{v_{e}}$ & $V V V$ & 0 & +1 & 0 \\
$d$ & $\bar{T} V V$ & $-\frac{1}{3}$ & $+\frac{1}{3}$ & 0 \\
$\bar{u}$ & $\bar{T} \bar{T} V$ & $-\frac{2}{3}$ & $-\frac{1}{3}$ & 0 \\
$e^{-}$ & $\bar{T} \bar{T} \bar{T}$ & -1 & -1 & 0 \\
\hline
\end{tabular}

Table 5. CGM of first generation of leptons and quarks

baryons (or antibaryons) of the SM. These leptons are built out of $T$ - and $V$-rishons or their antiparticles $\bar{T}$ and $\bar{V}$, all of which have generation quantum number $g=0$.

It is envisaged that each lepton of the first generation exists in an antisymmetric three-particle color state, which physically assumes a quantum mechanical triangular distribution of the three differently colored identical rishons (or antirishons), since each of the three color interactions between pairs of rishons (or antirishons) is expected to be strongly attractive (Halzen and Martin, 1984).

In the CGM, it is assumed that each quark of the first generation is a composite of a colored rishon and a colorless rishon-antirishon pair, $(T \bar{V})$ or $(V \bar{T})$, so that the quarks carry a color charge. Similarly, the antiquarks are a composite of an anticolored antirishon and a colorless rishon-antirishon pair, so that the antiquarks carry an anticolor charge.

In order to preserve the universality of the CC weak interaction processes involving first generation quarks, e.g. the transition $d \rightarrow u+W^{-}$, it is assumed that the first generation quarks have the general color structures:

$$
\text { up quark: } T_{C}\left(T_{C^{\prime}} \bar{V}_{\bar{C}^{\prime}}\right) \text {, down quark: } V_{C}\left(V_{C^{\prime}} \bar{T}_{\bar{C}^{\prime}}\right) \text {, with } C^{\prime} \neq C \text {. }
$$

Thus a red $u$-quark and a red $d$-quark have the general color structures:

$$
u_{r}=T_{r}\left(T_{g} \bar{V}_{\bar{g}}+T_{b} \bar{V}_{\bar{b}}\right) / \sqrt{2},
$$

and

$$
d_{r}=V_{r}\left(V_{g} \bar{T}_{\bar{g}}+V_{b} \bar{T}_{\bar{b}}\right) / \sqrt{2},
$$

respectively. For $d_{r} \rightarrow u_{r}+W^{-}$, conserving color, one has the two transitions:

$$
V_{r} V_{g} \bar{T}_{\bar{g}} \rightarrow T_{r} T_{b} \bar{V}_{\bar{b}}+V_{r} V_{g} V_{b} \bar{T}_{\bar{r}} \bar{T}_{\bar{g}} \bar{T}_{\bar{b}}
$$

and

$$
V_{r} V_{b} \bar{T}_{\bar{b}} \rightarrow T_{r} T_{g} \bar{V}_{\bar{g}}+V_{r} V_{g} V_{b} \bar{T}_{\bar{r}} \bar{T}_{\bar{g}} \bar{T}_{\bar{b}}
$$

which take place with equal probabilities. In these transitions, the $W^{-}$boson is assumed to be a three $\bar{T}$-antirishon and a three $V$-rishon colorless composite particle with additive quantum numbers $Q=-1, p=g=0$. The corresponding $W^{+}$boson has the structure $\left[T_{r} T_{g} T_{b} \bar{V}_{\bar{r}} \bar{V}_{\bar{g}} \bar{V}_{\bar{b}}\right]$, 


\begin{tabular}{|lcccc|}
\hline particle & structure & $Q$ & $p$ & $g$ \\
\hline$\mu^{+}$ & $T T T \Pi$ & +1 & +1 & \pm 1 \\
$c$ & $T T \bar{V} \Pi$ & $+\frac{2}{3}$ & $+\frac{1}{3}$ & \pm 1 \\
$\bar{s}$ & $T \bar{V} \bar{V} \Pi$ & $+\frac{1}{3}$ & $-\frac{1}{3}$ & \pm 1 \\
$v_{\mu}$ & $\bar{V} \bar{V} \bar{V} \Pi$ & 0 & -1 & \pm 1 \\
$\bar{v}_{\mu}$ & $V V V \Pi$ & 0 & +1 & \pm 1 \\
$s$ & $\bar{T} V V \Pi$ & $-\frac{1}{3}$ & $+\frac{1}{3}$ & \pm 1 \\
$\bar{c}$ & $\bar{T} \bar{T} V \Pi$ & $-\frac{2}{3}$ & $-\frac{1}{3}$ & \pm 1 \\
$\mu^{-}$ & $\bar{T} \bar{T} \bar{T} \Pi$ & -1 & -1 & \pm 1 \\
\hline
\end{tabular}

Table 6. CGM of second generation of leptons and quarks

consisting of a colorless set of three $T$-rishons and a colorless set of three $\bar{V}$-antirishons with additive quantum numbers $Q=+1, p=g=0$ (Robson, 2005).

The rishon structures of the second generation particles are the same as the corresponding particles of the first generation plus the addition of a colorless rishon-antirishon pair, $\Pi$, where

$$
\Pi=[(\bar{U} V)+(\bar{V} U)] / \sqrt{2},
$$

which is a quantum mechanical mixture of $(\bar{U} V)$ and $(\bar{V} U)$, which have $Q=p=0$ but $g= \pm 1$, respectively. In this way, the pattern for the first generation is repeated for the second generation. Table 6 gives the CGM structures for the second generation of leptons and quarks.

It should be noted that for any given transition the generation quantum number is required to be conserved, although each particle of the second generation has two possible values of $g$. For example, the decay

$$
\mu^{-} \rightarrow v_{\mu}+W^{-}
$$

at the rishon level may be written

$$
\bar{T} \bar{T} \bar{T} \Pi \rightarrow \bar{V} \bar{V} \bar{V} \Pi+\bar{T} \bar{T} \bar{T} V V V,
$$

which proceeds via the two transitions:

$$
\bar{T} \bar{T} \bar{T}(\bar{U} V) \rightarrow \bar{V} \bar{V} \bar{V}(\bar{U} V)+\bar{T} \bar{T} \bar{T} V V V
$$

and

$$
\bar{T} \bar{T} \bar{T}(\bar{V} U) \rightarrow \bar{V} \bar{V} \bar{V}(\bar{V} U)+\bar{T} \bar{T} \bar{T} V V V,
$$

which take place with equal probabilities. In each case, the additional colorless rishon-antirishon pair, $(\bar{U} V)$ or $(\bar{V} U)$, essentially acts as a spectator during the CC weak interaction process.

The rishon structures of the third generation particles are the same as the corresponding particles of the first generation plus the addition of two rishon-antirishon pairs, which are a quantum mechanical mixture of $(\bar{U} V)$ and $(\bar{V} U)$ and, as for the second generation, are assumed to be colorless and have $Q=p=0$ but $g= \pm 1$. In this way the pattern of the first and second generation is continued for the third generation. Table 7 gives the CGM structures for the third generation of leptons and quarks. 


\begin{tabular}{|lcccc|}
\hline particle & structure & $Q$ & $p$ & $g$ \\
\hline$\tau^{+}$ & $T T T \Pi \Pi$ & +1 & +1 & $0, \pm 2$ \\
$t$ & $T T \bar{V} \Pi \Pi$ & $+\frac{2}{3}$ & $+\frac{1}{3}$ & $0, \pm 2$ \\
$\bar{b}$ & $T \bar{V} \bar{V} \Pi \Pi$ & $+\frac{1}{3}$ & $-\frac{1}{3}$ & $0, \pm 2$ \\
$v_{\tau}$ & $\bar{V} \bar{V} \bar{V} \Pi \Pi$ & 0 & -1 & $0, \pm 2$ \\
$\bar{v}_{\tau}$ & $V V V \Pi \Pi$ & 0 & +1 & $0, \pm 2$ \\
$b$ & $\bar{T} V V \Pi \Pi$ & $-\frac{1}{3}$ & $+\frac{1}{3}$ & $0, \pm 2$ \\
$\bar{t}$ & $\bar{T} \bar{T} V \Pi \Pi$ & $-\frac{2}{3}$ & $-\frac{1}{3}$ & $0, \pm 2$ \\
$\tau^{-}$ & $\bar{T} \bar{T} \Pi \Pi \Pi$ & -1 & -1 & $0, \pm 2$ \\
\hline
\end{tabular}

Table 7. CGM of third generation of leptons and quarks

The rishon structure of the $\tau^{+}$particle is

$$
\operatorname{TTT\Pi \Pi ~}=\operatorname{TTT}[(\bar{U} V)(\bar{U} V)+(\bar{U} V)(\bar{V} U)+(\bar{V} U)(\bar{U} V)+(\bar{V} U)(\bar{V} U)] / 2
$$

and each particle of the third generation is a similar quantum mechanical mixture of $g=$ $0, \pm 2$ components. The color structures of both second and third generation leptons and quarks have been chosen so that the $\mathrm{CC}$ weak interactions are universal. In each case, the additional colorless rishon-antirishon pairs, $(\bar{U} V)$ and/or $(\bar{V} U)$, essentially act as spectators during any CC weak interaction process. Again it should be noted that for any given transition the generation quantum number is required to be conserved, although each particle of the third generation now has three possible values of $g$. Furthermore, in the CGM the three independent additive quantum numbers, charge $Q$, particle number $p$ and generation quantum number $g$, which are conserved in all interactions, correspond to the conservation of each of the three kinds of rishons (Robson, 2005):

$$
\begin{array}{r}
n(T)-n(\bar{T})=3 Q, \\
n(\bar{U})-n(\mathcal{U})=g, \\
n(T)+n(V)+n(U)-n(\bar{T})-n(\bar{V})-n(\bar{U})=3 p,
\end{array}
$$

where $n(R)$ and $n(\bar{R})$ are the numbers of rishons and antirishons, respectively. Thus, the conservation of $g$ in weak interactions is a consequence of the conservation of the three kinds of rishons ( $T, V$ and $U$ ), which also prohibits transitions between the third generation and the first generation via weak interactions even for $g=0$ components of third generation particles.

\subsection{Fundamental interactions of the GM}

The GM recognizes only two fundamental interactions in nature: (i) the usual electromagnetic interaction and (ii) a strong color-type interaction, mediated by massless hypergluons, acting between color charged rishons and/or antirishons.

The only essential difference between the strong color interactions of the GM and the SM is that the former acts between color charged rishons and/or antirishons while the latter acts between color charged elementary quarks and/or antiquarks. For historical reasons we use the term "hypergluons" for the mediators of the strong color interactions at the rishon level, rather than the term "gluons" as employed in the SM, although the effective color interaction between composite quarks and/or composite antiquarks is very similar to that between the elementary quarks and/or elementary antiquarks of the SM. 
In the GM both gravity and the weak interactions are considered to be residual interactions of the strong color interactions. Gravity will be discussed in some detail in Subsection 4.3. In the GM the weak interactions are assumed to be mediated by composite massive vector bosons, consisting of colorless sets of three rishons and three antirishons as discussed in the previous subsection, so that they are not elementary particles, associated with a $U(1) \times S U(2)_{L}$ local gauge theory as in the SM. The weak interactions are simply residual interactions of the CGM strong color force, which binds rishons and antirishons together, analogous to the strong nuclear interactions, mediated by massive mesons, being residual interactions of the strong color force of the SM, which binds quarks and antiquarks together. Since the weak interactions are not considered to be fundamental interactions arising from a local gauge theory, there is no requirement for the existence of a Higgs field to generate the boson masses within the framework of the GM (Robson, 2008).

\section{Consequences}

In this section it will be shown that new paradigms arising from the GM provide some understanding concerning: (i) the origin of mass; (ii) the mass hierarchy of leptons and quarks; (iii) the origin of gravity and (iv) the origin of "apparent" CP violation in the $K^{0}-\bar{K}^{0}$ system.

\subsection{Origin of mass}

Einstein (1905) concluded that the mass of a body $m$ is a measure of its energy content $E$ and is given by

$$
m=E / c^{2}
$$

where $c$ is the speed of light in a vacuum. This relationship was first tested by Cockcroft and Walton (1932) using the nuclear transformation

$$
{ }^{7} \mathrm{Li}+p \rightarrow 2 \alpha+17.2 \mathrm{MeV}
$$

and it was found that the decrease in mass in this disintegration process was consistent with the observed release of energy, according to Eq. (40). Recently, relation (40) has been verified (Rainville et al., 2005) to within $0.00004 \%$, using very accurate measurements of the atomic-mass difference, $\Delta m$, and the corresponding $\gamma$-ray wavelength to determine $E$, the nuclear binding energy, for isotopes of silicon and sulfur.

It has been emphasized by Wilczek (2005) that approximate QCD calculations (Butler et al., 1993; Aoki et al., 2000; Davies et al., 2004) obtain the observed masses of the neutron, proton and other baryons to an accuracy of within $10 \%$. In these calculations, the assumed constituents, quarks and gluons, are taken to be massless. Wilczek concludes that the calculated masses of the hadrons arise from both the energy stored in the motion of the quarks and the energy of the gluon fields, according to Eq. (40): basically the mass of a hadron arises from internal energy.

Wilzcek (2005) has also discussed the underlying principles giving rise to the internal energy, hence the mass, of a hadron. The nature of the gluon color fields is such that they lead to a runaway growth of the fields surrounding an isolated color charge. In fact all this structure (via virtual gluons) implies that an isolated quark would have an infinite energy associated with it. This is the reason why isolated quarks are not seen. Nature requires these infinities 
to be essentially cancelled or at least made finite. It does this for hadrons in two ways: either by bringing an antiquark close to a quark (i.e forming a meson) or by bringing three quarks, one of each color, together (i.e. forming a baryon) so that in each case the composite hadron is colorless. However, quantum mechanics prevents the quark and the antiquark of opposite colors or the three quarks of different colors from being placed exactly at the same place. This means that the color fields are not exactly cancelled, although sufficiently it seems to remove the infinities associated with isolated quarks. The distribution of the quark-antiquark pairs or the system of three quarks is described by quantum mechanical wave functions. Many different patterns, corresponding to the various hadrons, occur. Each pattern has a characteristic energy, because the color fields are not entirely cancelled and because the quarks are somewhat localized. This characteristic energy, E, gives the characteristic mass, via Eq. (40), of the hadron.

The above picture, within the framework of the SM, provides an understanding of hadron masses as arising mainly from internal energies associated with the strong color interactions. However, as discussed in Subsection 2.2.3, the masses of the elementary particles of the SM, the leptons, the quarks and the $W$ and $Z$ bosons, are interpreted in a completely different way. A "condensate" called the Higgs scalar field (Englert and Brout, 1964; Higgs, 1964), analogous to the Cooper pairs in a superconducting material, is assumed to exist. This field couples, with an appropriate strength, to each lepton, quark and vector boson and endows an originally massless particle with its physical mass. Thus, the assumption of a Higgs field within the framework of the SM not only adds an extra field but also leads to the introduction of 14 new parameters. Moreover, as pointed out by Lyre (2008), the introduction of the Higgs field in the $\mathrm{SM}$ to spontaneously break the $U(1) \times S U(2)_{L}$ local gauge symmetry of the electroweak interaction to generate the masses of the $W$ and $Z$ bosons, simply corresponds mathematically to putting in "by hand" the masses of the elementary particles of the SM: the so-called Higgs mechanism does not provide any physical explanation for the origin of the masses of the leptons, quarks and the $W$ and $Z$ bosons.

In the CGM (Robson, 2005; 2011a), the elementary particles of the SM have a substructure, consisting of massless rishons and/or antirishons bound together by strong color interactions, mediated by massless neutral hypergluons. This model is very similar to that of the SM in which the quarks and/or antiquarks are bound together by strong color interactions, mediated by massless neutral gluons, to form hadrons. Since, as discussed above, the mass of a hadron arises mainly from the energy of its constituents, the CGM suggests (Robson, 2009) that the mass of a lepton, quark or vector boson arises entirely from the energy stored in the motion of its constituent rishons and/or antirishons and the energy of the color hypergluon fields, E, according to Eq. (40). A corollary of this idea is: if a particle has mass, then it is composite. Thus, unlike the SM, the GM provides a unified description of the origin of all mass.

\subsection{Mass hierarchy of leptons and quarks}

Table 8 shows the observed masses of the charged leptons together with the estimated masses of the quarks: the masses of the neutral leptons have not yet been determined but are known to be very small. Although the mass of a single quark is a somewhat abstract idea, since quarks do not exist as particles independent of the environment around them, the masses of the quarks may be inferred from mass differences between hadrons of similar composition. The strong binding within hadrons complicates the issue to some extent but rough estimates of the quark masses have been made (Veltman, 2003), which are sufficient for our purposes. 


\begin{tabular}{|lcccc|}
\hline Charge & 0 & -1 & $+\frac{2}{3}$ & $-\frac{1}{3}$ \\
\hline Generation 1 & $v_{e}$ & $e^{-}$ & $u$ & $d$ \\
Mass & $<0.3 \mathrm{eV}$ & $0.511 \mathrm{MeV}$ & $5 \mathrm{MeV}$ & $10 \mathrm{MeV}$ \\
\hline Generation 2 & $v_{\mu}$ & $\mu^{-}$ & $c$ & $s$ \\
Mass & $<0.3 \mathrm{eV}$ & $106 \mathrm{MeV}$ & $1.3 \mathrm{GeV}$ & $200 \mathrm{MeV}$ \\
\hline Generation 3 & $v_{\tau}$ & $\tau^{-}$ & $t$ & $b$ \\
Mass & $<0.3 \mathrm{eV}$ & $1.78 \mathrm{GeV}$ & $175 \mathrm{GeV}$ & $4.5 \mathrm{GeV}$ \\
\hline
\end{tabular}

Table 8. Masses of leptons and quarks

The SM is unable to provide any understanding of either the existence of the three generations of leptons and quarks or their mass hierarchy indicated in Table 8; whereas the CGM suggests that both the existence and mass hierarchy of these three generations arise from the substructures of the leptons and quarks (Robson, 2009; 2011a).

Subsection 3.3 describes the proposed rishon and/or antirishon substructures of the three generations of leptons and quarks and indicates how the pattern of the first generation is followed by the second and third generations. Section 4.1 discusses the origin of mass in composite particles and postulates that the mass of a lepton or quark arises from the energy of its constituents.

In the CGM it is envisaged that the rishons and/or antirishons of each lepton or quark are very strongly localized, since to date there is no direct evidence for any substructure of these particles. Thus the constituents are expected to be distributed according to quantum mechanical wave functions, for which the product wave function is significant for only an extremely small volume of space so that the corresponding color fields are almost cancelled. The constituents of each lepton or quark are localized within a very small volume of space by strong color interactions acting between the colored rishons and/or antirishons. We call these intra-fermion color interactions. However, between any two leptons and/or quarks there will be a residual interaction, arising from the color interactions acting between the constituents of one fermion and the constituents of the other fermion. We refer to these interactions as inter-fermion color interactions. These will be associated with the gravitational interaction and are discussed in the next subsection.

The mass of each lepton or quark corresponds to a characteristic energy primarily associated with the intra-fermion color interactions. It is expected that the mass of a composite particle will be greater if the degree of localization of its constituents is smaller (i.e. the constituents are on average more widely separated). This is a consequence of the nature of the strong color interactions, which are assumed to possess the property of "asymptotic freedom" (Gross and Wilczek, 1973; Politzer, 1973), whereby the color interactions become stronger for larger separations of the color charges. In addition, it should be noted that the electromagnetic interactions between charged $T$-rishons or between charged $\bar{T}$-antirishons will also cause the degree of localization of the constituents to be smaller causing an increase in mass.

There is some evidence for the above expectations. The electron consists of three $\bar{T}$-antirishons, while the electron neutrino consists of three neutral $\bar{V}$-antirishons. Neglecting the electric charge carried by the $\bar{T}$-antirishon, it is expected that the electron and its neutrino would have identical masses, arising from the similar intra-fermion color interactions. However, it is anticipated that the electromagnetic interaction in the electron case will cause the $\bar{T}$-antirishons to be less localized than the $\bar{V}$-antirishons constituting the electron neutrino 
so that the electron will have a substantially greater characteristic energy and hence a greater mass than the electron neutrino, as observed. This large difference in the masses of the $e^{-}$ and $v_{e}$ leptons (see Table 8) indicates that the mass of a particle is extremely sensitive to the degree of localization of its constituents. Similarly, the up, charmed and top quarks, each containing two charged $T$-rishons, are expected to have a greater mass than their weak isospin partners, the down, strange and bottom quark, respectively, which contain only a single charged $\bar{T}$-antirishon. This is true provided one takes into account quark mixing (Evans and Robson, 2006) in the case of the up and down quarks, although Table 8 indicates that the down quark is more massive than the up quark, leading to the neutron having a greater mass than the proton. This is understood within the framework of the GM since due to the manner in which quark masses are estimated, it is the weak eigenstate quarks, whose masses are given in Table 8. Since each succeeding generation is significantly more massive than the previous one, any mixing will noticeably increase the mass of a lower generation quark. Thus the weak eigenstate $d^{\prime}$-quark, which contains about $5 \%$ of the mass eigenstate $s$-quark, is expected to be significantly more massive than the mass eigenstate $d$-quark (see Subsection 3.2). We shall now discuss the mass hierarchy of the three generations of leptons and quarks in more detail.

It is envisaged that each lepton of the first generation exists in an antisymmetric three-particle color state, which physically assumes a quantum mechanical triangular distribution of the three differently colored identical rishons (or antirishons) since each of the three color interactions between pairs of rishons (or antirishons) is expected to be strongly attractive (Halzen and Martin, 1984). As indicated above, the charged leptons are predicted to have larger masses than the neutral leptons, since the electromagnetic interaction in the charged leptons will cause their constituent rishons (or antirishons) to be less localized than those constituting the uncharged leptons, leading to a substantially greater characteristic energy and a correspondingly greater mass.

In the CGM, each quark of the first generation is a composite of a colored rishon and a colorless rishon-antirishon pair, $(T \bar{V})$ or a $(V \bar{T})$ (see Table 5). This color charge structure of the quarks is expected to lead to a quantum mechanical linear distribution of the constituent rishons and antirishons, corresponding to a considerably larger mass than that of the leptons, since the constituents of the quarks are less localized. This is a consequence of the character (i.e. attractive or repulsive) of the color interactions at small distances (Halzen and Martin, 1984). The general rules for small distances of separation are:

(i) rishons (or antirishons) of like colors (or anticolors) repel: those having different colors (or anticolors) attract, unless their colors (or anticolors) are interchanged and the two rishons (or antirishons) do not exist in an antisymmetric color state (e.g. as in the case of leptons);

(ii) rishons and antirishons of opposite colors attract but otherwise repel.

Furthermore, the electromagnetic interaction occurring within the up quark, leads one to expect it to have a larger mass than that of the down quark.

Each lepton of the second generation is envisaged to basically exist in an antisymmetric three-particle color state, which physically assumes a quantum mechanical triangular distribution of the three differently colored identical rishons (or antirishons), as for the corresponding lepton of the first generation. The additional colorless rishon-antirishon pair, $(V \bar{U})$ or $(U \bar{V})$, is expected to be attached externally to this triangular distribution, leading quantum mechanically to a less localized distribution of the constituent rishons and/or 
antirishons, so that the lepton has a significantly larger mass than its corresponding first generation lepton.

Each quark of the second generation has a similar structure to that of the corresponding quark of the first generation, with the additional colorless rishon-antirishon pair, $(V \bar{U})$ or $(U \bar{V})$, attached quantum mechanically so that the whole rishon structure is essentially a linear distribution of the constituent rishons and antirishons. This structure is expected to be less localized, leading to a larger mass relative to that of the corresponding quark of the first generation, with the charmed quark having a greater mass than the strange quark, arising from the electromagnetic repulsion of its constituent two charged $T$-rishons.

Each lepton of the third generation is considered to basically exist in an antisymmetric three-particle color state, which physically assumes a quantum mechanical triangular distribution of the three differently colored identical rishons (or antirishons), as for the corresponding leptons of the first and second generations. The two additional colorless rishon-antirishon pairs, $(V \bar{U})(V \bar{U}),(V \bar{U})(U \bar{V})$ or $(U \bar{V})(U \bar{V})$, are expected to be attached externally to this triangular distribution, leading to a considerably less localized quantum mechanical distribution of the constituent rishons and/or antirishons, so that the lepton has a significantly larger mass than its corresponding second generation lepton.

Each quark of the third generation has a similar structure to that of the first generation, with the additional two rishon-antirishon pairs $(V \bar{U})$ and/or $(U \bar{V})$ attached quantum mechanically so that the whole rishon structure is essentially a linear distribution of the constituent rishons and antirishons. This structure is expected to be even less localized, leading to a larger mass relative to that of the corresponding quark of the second generation, with the top quark having a greater mass than the bottom quark, arising from the electromagnetic repulsion of its constituent two charged T-rishons.

The above is a qualitative description of the mass hierarchy of the three generations of leptons and quarks, based on the degree of localization of their constituent rishons and/or antirishons. However, in principle, it should be possible to calculate the actual masses of the leptons and quarks by carrying out QCD-type computations, analogous to those employed for determining the masses of the proton and other baryons within the framework of the SM (Butler et al., 1993; Aoki et al., 2000; Davies et al., 2004).

\subsection{Origin of gravity}

Robson (2009) proposed that the residual interaction, arising from the incomplete cancellation of the inter-fermion color interactions acting between the rishons and/or antirishons of one colorless particle and those of another colorless particle, may be identified with the usual gravitational interaction, since it has several properties associated with that interaction: universality, infinite range and very weak strength. Based upon this earlier conjecture, Robson (2011a) has presented a quantum theory of gravity, described below, leading approximately to Newton's law of universal gravitation.

The mass of a body of ordinary matter is essentially the total mass of its constituent electrons, protons and neutrons. It should be noted that these masses will depend upon the environment in which the particle exists: e.g. the mass of a proton in an atom of helium will differ slightly from that of a proton in an atom of lead. In the CGM, each of these three particles is considered 
to be colorless. The electron is composed of three $\bar{T}$-antirishons, each carrying a different anticolor charge, antired, antigreen or antiblue. Both the proton and neutron are envisaged (as in the SM) to be composed of three quarks, each carrying a different color charge, red, green or blue. All three particles are assumed to be essentially in a three-color antisymmetric state, so that their behavior with respect to the strong color interactions is expected basically to be the same. This similar behavior suggests that the proposed residual interaction has several properties associated with the usual gravitational interaction.

Firstly, the residual interaction between any two of the above colorless particles, arising from the inter-fermion color interactions, is predicted to be of a universal character.

Secondly, assuming that the strong color fields are almost completely cancelled at large distances, it seems plausible that the residual interaction, mediated by massless hypergluons, should have an infinite range, and tend to zero as $1 / r^{2}$. These properties may be attributed to the fact that the constituents of each colorless particle are very strongly localized so that the strength of the residual interaction is extremely weak, and consequently the hypergluon self-interactions are also practically negligible. This means that one may consider the color interactions using a perturbation approach: the residual color interaction is the sum of all the two-particle color charge interactions, each of which may be treated perturbatively, i.e. as a single hypergluon exchange. Using the color factors (Halzen and Martin, 1984) appropriate for the $S U(3)$ gauge field, one finds that the residual color interactions between any two colorless particles (electron, neutron or proton) are each attractive.

Since the mass of a body of ordinary matter is essentially the total mass of its constituent electrons, neutrons and protons, the total interaction between two bodies of masses, $m_{1}$ and $m_{2}$, will be the sum of all the two-particle contributions so that the total interaction will be proportional to the product of these two masses, $m_{1} m_{2}$, provided that each two-particle interaction contribution is also proportional to the product of the masses of the two particles.

This latter requirement may be understood if each electron, neutron or proton is considered physically to be essentially a quantum mechanical triangular distribution of three differently colored rishons or antirishons. In this case, each particle may be viewed as a distribution of three color charges throughout a small volume of space with each color charge having a certain probability of being at a particular point, determined by its corresponding color wave function. The total residual interaction between two colorless particles will then be the sum of all the intrinsic interactions acting between a particular triangular distribution of one particle with that of the other particle.

Now the mass $m$ of each colorless particle is considered to be given by $m=E / c^{2}$, where $E$ is a characteristic energy, determined by the degree of localization of its constituent rishons and/or antirishons. Thus the significant volume of space occupied by the triangular distribution of the three differently colored rishons or antirishons is larger the greater the mass of the particle. Moreover, due to antiscreening effects (Gross and Wilczek, 1973; Politzer, 1973) of the strong color fields, the average strength of the color charge within each unit volume of the larger localized volume of space will be increased. If one assumes that the mass of a particle is proportional to the integrated sum of the intra-fermion interactions within the significant volume of space occupied by the triangular distribution, then the total residual interaction between two such colorless particles will be proportional to the product of their masses. 
Thus the residual color interaction between two colorless bodies of masses, $m_{1}$ and $m_{2}$, is proportional to the product of these masses and moreover is expected to depend approximately as the inverse square of their distance of separation $r$, i.e. as $1 / r^{2}$, in accordance with Newton's law of universal gravitation. The approximate dependence on the inverse square law is expected to arise from the effect of hypergluon self-interactions, especially for large separations. Such deviations from an inverse square law do not occur for electromagnetic interactions, since there are no corresponding photon self-interactions.

\subsection{Mixed-quark states in hadrons}

As discussed in Subsection 3.2 the GM postulates that hadrons are composed of weak eigenstate quarks rather than mass eigenstate quarks as in the SM. This gives rise to several important consequences (Evans and Robson, 2006; Morrison and Robson, 2009; Robson, 2011b; 2011c).

Firstly, hadrons composed of mixed-quark states might seem to suggest that the electromagnetic and strong interaction processes between mass eigenstate hadron components are not consistent with the fact that weak interaction processes occur between weak eigenstate quarks. However, since the electromagnetic and strong interactions are flavor independent: the down, strange and bottom quarks carry the same electric and color charges so that the weak eigenstate quarks have the same magnitude of electric and color charge as the mass eigenstate quarks. Consequently, the weak interaction is the only interaction in which the quark-mixing phenomenon can be detected.

Secondly, the occurrence of mixed-quark states in hadrons implies the existence of higher generation quarks in hadrons. In particular, the GM predicts that the proton contains $\approx 1.7 \%$ of strange quarks, while the neutron having two $d^{\prime}$-quarks contains $\approx 3.4 \%$ of strange quarks. Recent experiments (Maas et al., 2005; Armstrong et al, 2005) have provided some evidence for the existence of strange quarks in the proton. However, to date the experimental data are compatible with the predictions of both the GM and the SM $(\ll 1.7 \%)$.

Thirdly, the presence of strange quarks in nucleons explains why the mass of the neutron is greater than the mass of a proton, so that the proton is stable. This arises because the mass of the weak eigenstate $d^{\prime}$-quark is larger than the mass of the $u$-quark, although the mass eigenstate $d$-quark is expected to be smaller than that of the $u$-quark, as discussed in the previous section.

Another consequence of the presence of mixed-quark states in hadrons is that mixed-quark states may have mixed parity. In the CGM the constituents of quarks are rishons and/or antirishons. If one assumes the simple convention that all rishons have positive parity and all their antiparticles have negative parity, one finds that the down and strange quarks have opposite intrinsic parities, according to the proposed structures of these quarks in the CGM: the $d$-quark (see Table 5) consists of two rishons and one antirishon $\left(P_{d}=-1\right)$, while the $s$-quark (see Table 6) consists of three rishons and two antirishons $\left(P_{s}=+1\right)$. The $u$-quark consists of two rishons and one antirishon so that $P_{u}=-1$, and the antiparicles of these three quarks have the corresponding opposite parities: $P_{\bar{d}}=+1, P_{\bar{s}}=-1$ and $P_{\bar{u}}=+1$.

In the SM the intrinsic parity of the charged pions is assumed to be $P_{\pi}=-1$. This result was established by Chinowsky and Steinberger (1954), using the capture of negatively charged pions in deuterium to form two neutrons, and led to the overthrow of the conservation of 
both parity (P) and charge-conjugation (C) (Lee and Yang, 1956; Wu et al., 1957; Garwin et al., 1957; Friedman and Telegdi, 1957) and later combined CP conservation (Christenson et al., 1964). Recently, Robson (2011b) has demonstrated that this experiment is also compatible with the mixed-parity nature of the $\pi^{-}$predicted by the CGM: $\approx\left(0.95 P_{d}+0.05 P_{s}\right)$, with $P_{d}=-1$ and $P_{S}=+1$. This implies that the original determination of the parity of the negatively charged pion is not conclusive, if the pion has a complex substructure as in the CGM. Similarly, Robson (2011c) has shown that the recent determination (Abouzaid et al., 2008) of the parity of the neutral pion, using the double Dalitz decay $\pi^{0} \rightarrow e^{+} e^{-} e^{+} e^{-}$is also compatible with the mixed-parity nature of the neutral pion predicted by the CGM.

This new concept of mixed-parity states in hadrons, based upon the existence of weak eigenstate quarks in hadrons and the composite nature of the mass eigenstate quarks, leads to an understanding of CP symmetry in nature. This is discussed in the following subsection.

\subsection{CP violation in the $K^{0}-\bar{K}^{0}$ system}

Gell-Mann and Pais (1955) considered the behavior of neutral particles under the charge-conjugation operator $C$. In particular they considered the $K^{0}$ meson and realized that unlike the photon and the neutral pion, which transform into themselves under the Coperator so that they are their own antiparticles, the antiparticle of the $K^{0}$ meson (strangeness $S=+1$ ), $\bar{K}^{0}$, was a distinct particle, since it had a different strangeness quantum number $(S=-1)$. They concluded that the two neutral mesons, $K^{0}$ and $\bar{K}^{0}$, are degenerate particles that exhibit unusual properties, since they can transform into each other via weak interactions such as

$$
K^{0} \rightleftharpoons \pi^{+} \pi^{-} \rightleftharpoons \bar{K}^{0} .
$$

In order to treat this novel situation, Gell-Mann and Pais suggested that it was more convenient to employ different particle states, rather than $K^{0}$ and $\bar{K}^{0}$, to describe neutral kaon decay. They suggested the following representative states:

$$
K_{1}^{0}=\left(K^{0}+\bar{K}^{0}\right) / \sqrt{2}, \quad K_{2}^{0}=\left(K^{0}-\bar{K}^{0}\right) / \sqrt{2},
$$

and concluded that these particle states must have different decay modes and lifetimes. In particular they concluded that $K_{1}^{0}$ could decay to two charged pions, while $K_{2}^{0}$ would have a longer lifetime and more complex decay modes. This conclusion was based upon the conservation of $C$ in the weak interaction processes: both $K_{1}^{0}$ and the $\pi^{+} \pi^{-}$system are even (i.e. $C=+1$ ) under the $C$ operation.

The particle-mixing theory of Gell-Mann and Pais was confirmed in 1957 by experiment, in spite of the incorrect assumption of $C$ invariance in weak interaction processes. Following the discovery in 1957 of both $\mathrm{C}$ and $\mathrm{P}$ violation in weak interaction processes, the particle-mixing theory led to a suggestion by Landau (1957) that the weak interactions may be invariant under the combined operation $\mathrm{CP}$.

Landau's suggestion implied that the Gell-Mann-Pais model of neutral kaons would still apply if the states, $K_{1}^{0}$ and $K_{2}^{0}$, were eigenstates of $\mathrm{CP}$ with eigenvalues +1 and -1 , respectively. Since the charged pions were considered to have intrinsic parity $P_{\pi}=-1$, it was clear that only the $K_{1}^{0}$ state could decay to two charged pions, if CP was conserved.

The suggestion of Landau was accepted for several years since it nicely restored some degree of symmetry in weak interaction processes. However, the surprising discovery (Christenson 
et al., 1964) of the decay of the long-lived neutral $K^{0}$ meson to two charged pions led to the conclusion that $\mathrm{CP}$ is violated in the weak interaction. The observed violation of $\mathrm{CP}$ conservation turned out to be very small $(\approx 0.2 \%)$ compared with the maximal violations $(\approx$ $100 \%)$ of both P and C conservation separately. Indeed the very smallness of the apparent $\mathrm{CP}$ violation led to a variety of suggestions explaining it in a $\mathrm{CP}$-conserving way (Kabir, 1968; Franklin, 1986). However, these efforts were unsuccessful and CP violation in weak interactions was accepted.

An immediate consequence of this was that the role of $K_{1}^{0}(\mathrm{CP}=+1)$ and $K_{2}^{0}(\mathrm{CP}=-1)$, defined in Eqs. (43), was replaced by two new particle states, corresponding to the short-lived $\left(K_{S}^{0}\right)$ and long-lived $\left(K_{L}^{0}\right)$ neutral kaons:

$$
K_{S}^{0}=\left(K_{1}^{0}+\epsilon K_{2}^{0}\right) /\left(1+|\epsilon|^{2}\right)^{\frac{1}{2}}, \quad K_{L}^{0}=\left(K_{2}^{0}+\epsilon K_{1}^{0}\right) /\left(1+|\epsilon|^{2}\right)^{\frac{1}{2}},
$$

where the small complex parameter $\epsilon$ is a measure of the CP impurity in the eigenstates $K_{S}^{0}$ and $K_{L}^{0}$. This method of describing CP violation in the Standard Model (SM), by introducing mixing of CP eigenstates, is called 'indirect CP violation'. It is essentially a phenomenological approach with the parameter $\epsilon$ to be determined by experiment.

Another method of introducing CP violation into the SM was proposed by Kobayashi and Maskawa (1973). By extending the idea of 'Cabibbo mixing' (see Subsection 2.2.3) to three generations, they demonstrated that this allowed a complex phase to be introduced into the quark-mixing (CKM) matrix, permitting $\mathrm{CP}$ violation to be directly incorporated into the weak interaction. This phenomenological method has within the framework of the SM successfully accounted for both the indirect CP violation discovered by Christenson et al. in 1964 and the "direct CP violation" related to the decay processes of the neutral kaons (Kleinknecht, 2003). However, to date, the phenomenological approach has not been able to provide an a priori reason for $\mathrm{CP}$ violation to occur nor to indicate the magnitude of any such violation.

Recently, Morrison and Robson (2009) have demonstrated that the indirect CP violation observed by Christenson et al. (1964) can be described in terms of mixed-quark states in hadrons. In addition, the rate of the decay of the $K_{L}^{0}$ meson relative to the decay into all charged modes is estimated accurately in terms of the Cabibbo-mixing angle.

In the CGM the $K^{0}$ and $\bar{K}^{0}$ mesons have the weak eigenstate quark structures $\left[d^{\prime} \bar{s}^{\prime}\right]$ and $\left[s^{\prime} \bar{d}^{\prime}\right]$, respectively. Neglecting the very small mixing components arising from the third generation, Morrison and Robson show that the long-lived neutral kaon, $K_{L}^{0}$, exists in a $\mathrm{CP}=-1$ eigenstate as in the SM. On the other hand, the charged $2 \pi$ system:

$$
\begin{aligned}
\pi^{+} \pi^{-}= & {\left[u \bar{d}^{\prime}\right]\left[d^{\prime} \bar{u}\right] } \\
= & {[u \bar{d}][d \bar{u}] \cos ^{2} \theta_{c}+[u \bar{s}][s \bar{u}] \sin ^{2} \theta_{c}+[u \bar{s}][d \bar{u}] \sin \theta_{c} \cos \theta_{c} } \\
& +[u \bar{d}][s \bar{u}]) \sin \theta_{c} \cos \theta_{c} .
\end{aligned}
$$

For the assumed parities (see Subsection 4.4) of the quarks and antiquarks involved in Eq. (45), it is seen that the first two components are eigenstates of $C P=+1$, while the remaining two components $[u \bar{s}][d \bar{u}]$ and $[u \bar{d}][s \bar{u}]$, with amplitude $\sin \theta_{c} \cos \theta_{c}$ are not individually eigenstates of CP. However, taken together, the state $([u \bar{s}][d \bar{u}]+[u \bar{d}][s \bar{u}])$ is an eigenstate of CP with eigenvalue $\mathrm{CP}=-1$. Taking the square of the product of the amplitudes of the two components comprising the $\mathrm{CP}=-1$ eigenstate to be the "joint probability" of those two states existing 
together simultaneously, one can calculate that this probability is given by $\left(\sin \theta_{c} \cos \theta_{c}\right)^{4}$ $=2.34 \times 10^{-3}$, using $\cos \theta_{c}=0.9742$ (Amsler et al., 2008). Thus, the existence of a small component of the $\pi^{+} \pi^{-}$system with eigenvalue $C P=-1$ indicates that the $K_{L}^{0}$ meson can decay to the charged $2 \pi$ system without violating $\mathrm{CP}$ conservation. Moreover, the estimated decay rate is in good agreement with experimental data (Amsler et al., 2008).

\section{Summary and future prospects}

The GM, which contains fewer elementary particles (27 counting both particles and antiparticles and their three different color forms) and only two fundamental interactions (the electromagnetic and strong color interactions), has been presented as a viable simpler alternative to the SM (61 elementary particles and four fundamental interactions).

In addition, the GM has provided new paradigms for particle physics, which have led to a new understanding of several phenomena not addressed by the SM. In particular, (i) the mass of a particle is attributed to the energy content of its constituents so that there is no requirement for the Higgs mechanism; (ii) the mass hierarchy of the three generations of leptons and quarks is described by the degree of localization of their constituent rishons and/or antirishons; (iii) gravity is interpreted as a quantum mechanical residual interaction of the strong color interaction, which binds rishons and/or antirishons together to form all kinds of matter and (iv) the decay of the long-lived neutral kaon is understood in terms of mixed-quark states in hadrons and not $\mathrm{CP}$ violation.

The GM also predicts that the mass of a free neutron is greater than the mass of a free proton so that the free proton is stable. In addition, the model predicts the existence of higher generation quarks in hadrons, which in turn predicts mixed-parity states in hadrons. Further experimentation is required to verify these predictions and thereby strengthen the Generation Model.

\section{References}

Abouzaid, E. et al. (2008), Determination of the Parity of the Neutral Pion via its Four-Electron Decay, Physical Review Letters, Vol. 100, No. 18, 182001 (5 pages).

Aitchison I.J.R. and Hey, A.J.G. (1982), Gauge Theories in Particle Physics (Adam Hilger Ltd, Bristol).

Amsler, C. et al. (2008), Summary Tables of Particle Properties, Physics Letters B, Vol. 667, Nos. $1-5$, pp. 31-100.

Aoki, S. et al. (2000), Quenched Light Hadron Spectrum, Physical Review Letters, Vol. 84, No. 2, pp. 238-241.

Armstrong, D.S. et al. (2005), Strange-Quark Contributions to Parity-Violating Asymmetries in the Forward G0 Electron-Proton Scattering Experiment, Physical Review Letters, Vol. 95, No. 9, 092001 (5 pages).

Arnison, G. et al. (1983), Experimental Observation of Isolated Large Transverse Energy Electrons with Associated Missing Energy, Physics Letters B, Vol 122, No. 1, pp. 103-116.

Banner, M. et al. (1983), Observation of Single Isolated Electrons of High Transverse Momentum in Events with Missing Transverse Energy at the CERN pp Collider, Physics Letters B, Vol. 122, Nos. 5-6, pp. 476-485. 
Bloom, E.D. et al. (1969), High-Energy Inelastic $e-p$ Scattering at $6^{0}$ and $10^{0}$, Physical Review Letters, Vol. 23, No. 16, pp. 930-934.

Breidenbach, M. et al. (1969), Observed Behavior of Highly Inelastic Electron-Proton Scattering, Physical Review Letters, Vol. 23, No. 16, pp. 935-939.

Butler, F. et al. (1993), Hadron Mass Predictions of the Valence Approximation to Lattice QCD, Physical Review Letters, Vol. 70, No. 19, pp. 2849-2852.

Cabibbo, N. (1963), Unitary Symmetry and Leptonic Decays, Physical Review Letters, Vol. 10, No. 12, pp. 531-533.

Casalbuoni, R. and Gatto, R. (1980), Subcomponent Models of Quarks and Leptons, Physics Letters B, Vol. 93, Nos. 1-2, pp. 47-52.

Chinowsky, W. and Steinberger, J. (1954), Absorption of Negative Pions in Deuterium: Parity of the Pion, Physical Review, Vol. 95, No. 6, pp. 1561-1564.

Christenson, J.H. et al. (1964), Evidence for the $2 \pi$ Decay of the $K_{2}^{0}$ Meson, Physical Review Letters, Vol. 13, No. 4, pp. 138-140.

Cockcroft, J. and Walton, E. (1932), Experiments with High Velocity Positive Ions. II. The Disintegration of Elements by High Velocity Protons, Proceedings of the Royal Society of London, Series A, Vol. 137, No. 831, pp. 239-242.

Davies, C.T.H. et al. (2004), High-Precision Lattice QCD Confronts Experiment, Physical Review Letters, Vol. 92, No. 2, 022001 (5 pages).

Einstein, A. (1905), Ist die Trägheit eines Körpers von seinem Energieinhalt abhängig, Annalen der Physik, Vol. 18, No. 13, pp. 639-641.

Englert, F. and Brout, R. (1964), Broken Symmetry and the Mass of Gauge Vector Bosons, Physical Review Letters, Vol. 13, No. 9, pp. 321-323.

Evans, P.W. and Robson, B.A. (2006), Comparison of Quark Mixing in the Standard and Generation Models, International Journal of Modern Physics E, Vol. 15, No 3, pp. 617-625.

Franklin, A. (1986), The Neglect of Experiment (Cambridge University Press, Cambridge, U.K.).

Friedman, J.I. and Telegdi, V.L. (1957), Nuclear Emulsion Evidence for Parity Nonconservation in the Decay Chain $\pi^{+}-\mu^{+}-e^{+}$, Physical Review, Vol. 105, No. 5, pp. 1681-1682.

Garwin, R.L., Lederman, L.M. and Weinrich, M. (1957), Observations of the Failure of Conservation of Parity and Charge Conjugation in Meson Decays: the Magnetic Moment of the Free Muon, Physical Review, Vol. 105, No. 4, pp. 1415-1417.

Gell-Mann, M. and Ne'eman, Y. (1964), The Eightfold Way, (Benjamin, New York).

Gell-Mann, M. and Pais, A. (1955), Behavior of Neutral Particles under Charge Conjugation, Physical Review, Vol. 97, No. 5, pp. 1387-1389.

Glashow, S.L. (1961), Partial-Symmetries of Weak Interactions, Nuclear Physics, Vol. 22, pp. 579-588.

Gottfried, K. and Weisskopf, V.F. (1984), Concepts of Particle Physics Vol. 1 (Oxford University Press, New York).

Gross, D.J. and Wilczek, F. (1973), Ultraviolet Behavior of Non-Abelian Gauge Theories, Physical Review Letters, Vol. 30, No. 26, pp. 1343-1346.

Halzen, F. and Martin, A.D. (1984), Quarks and Leptons: An Introductory Course in Modern Particle Physics (John Wiley and Sons, New York).

Harari, H. (1979), A Schematic Model of Quarks and Leptons, Physics Letters B, Vol. 86, No. 1, pp. 83-86.

Harari, H. and Seiberg, N. (1981), A Dynamical Theory for the Rishon Model, Physics Letters B, Vol.98, No. 4, pp. 269-273. 
Hasert et al. (1973), Observation of Neutrino-Like Interactions without Muon or Electron in the Gargamelle Neutrino Experiment, Physics Letters B, Vol. 46, No. 1, pp. 138-140.

Hasert et al. (1974), Observation of Neutrino-Like Interactions without Muon or Electron in the Gargamelle Neutrino Experiment, Nuclear Physics B, Vol. 73, No. 1, pp. 1-22.

Higgs, P.W. (1964), Broken Symmetries and the Masses of Gauge Bosons, Physical Review Letters, Vol. 13, No. 16, pp. 508-509.

Kabir, P.K. (1968), The CP Puzzle: Strange Decays of the Neutral Kaon (Academic Press, London).

Kleinknecht, K. (2003), Uncovering CP Violation: Experimental Clarification in the Neutral K Meson and B Meson Systems (Springer, Berlin).

Kobayashi, M. and Maskawa, T. (1973), CP-Violation in Renormalizable Theory of Weak Interaction, Progress of Theoretical Physics, Vol. 49, No. 2, pp. 652-657.

Landau, L.D. (1957), On the Conservation Laws for Weak Interactions, Nuclear Physics, Vol. 3 , No.1, pp. 127-131.

Lee, T.D. and Yang, C.N. (1956), Question of Parity Conservation in Weak Interactions, Physical Review, Vol. 104, No. 1, pp. 254-258.

Lyre, H. (2008), Does the Higgs Mechanism Exist?, International Studies in the Philosophy of Science, Vol. 22, No. 2, pp. 119-133.

Mass, F.E. et al., Evidence for Strange-Quark Contributions to the Nucleon's Form Factors at $\mathrm{Q}^{2}=0.108(\mathrm{GeV} / \mathrm{c})^{2}$, Physical Review Letters, Vol. 94, No. 15, 152001 (4 pages).

Morrison, A.D. and Robson, B.A. (2009), $2 \pi$ Decay of the $K_{L}^{0}$ Meson without CP Violation, International Journal of Modern Physics E, Vol. 18, No. 9, pp. 1825-1830.

Politzer, H.D. (1973), Reliable Perturbative Results for Strong Interactions, Physical Review Letters, Vol. 30, No. 26, pp. 1346-1349.

Rainville, S. et al. (2005), World Year of Physics: A Direct Test of $E=\mathrm{mc}^{2}$, Nature, Vol. 438, pp. 1096-1097.

Robson, B.A. (2002), A Generation Model of the Fundamental Particles, International Journal of Modern Physics E, Vol. 11, No. 6, pp. 555-566.

Robson, B.A. (2004), Relation between Strong and Weak Isospin, International Journal of Modern Physics E, Vol. 13, No. 5, pp. 999-1018.

Robson, B.A. (2005), A Generation Model of Composite Leptons and Quarks, International Journal of Modern Physics E, Vol. 14, No. 8, pp. 1151-1169.

Robson, B.A. (2008), The Generation Model and the Electroweak Connection, International Journal of Modern Physics E, Vol. 17, No. 6, pp. 1015-1030.

Robson, B.A. (2009), The Generation Model and the Origin of Mass, International Journal of Modern Physics E, Vol. 18, No. 8, pp. 1773-1780.

Robson, B.A. (2011a), A Quantum Theory of Gravity based on a Composite Model of Leptons and Quarks, International Journal of Modern Physics E, Vol. 20, No. 3, pp. 733-745.

Robson, B.A. (2011b), Parity of Charged Pions, International Journal of Modern Physics E, Vol. 20, No. 8, pp. 1677-1686.

Robson, B.A. (2011c), Parity of Neutral Pion, International Journal of Modern Physics E, Vol. 20, No. 9, pp. 1961-1965.

Salam, A. (1968) in Elementary Particle Physics (Proceedings of the 8th Nobel Symposium), ed. Svartholm, N. (Almqvist and Wiksell, Stockholm), p. 367.

Shupe, M.A. (1979), A Composite Model of Leptons and Quarks, Physics Letters B, Vol. 86, No. 1, pp. 87-92.

Squires, E.J. (1980), QDD-a Model of Quarks and Leptons, Physics Letters B, Vol. 94, No. 1, pp. 54-56. 
Squires, E.J. (1981), Some Comments on the Three-Fermion Composite Quark and Lepton Model, Journal of Physics G, Vol. 7, No. 4, pp. L47-L49.

t'Hooft, G. (1971a), Renormalization of Massless Yang-Mills Fields, Nuclear Physics B, Vol. 33, No. 1, pp. 173-199.

t'Hooft, G. (1971b), Renormalizable Lagrangians for Massive Yang-Mills Fields, Nuclear Physics B, Vol. 35, No. 1, pp. 167-188.

Veltman, M. (2003), Facts and Mysteries in Elementary Particle Physics, (World Scientific Publishing Company, Singapore).

Weinberg, S. (1967), A Model of Leptons, Physical Review Letters, Vol. 19, No. 21, pp. 1264-1266.

Wilczek, F. (2005) In Search of Symmetry Lost, Nature, Vol. 433, No. 3, pp. 239-247.

Wu, C.S. et al. (1957), Experimental Test of Parity Conservation in Beta Decay, Physical Review, Vol. 105, No. 4, pp. 1413-1415. 


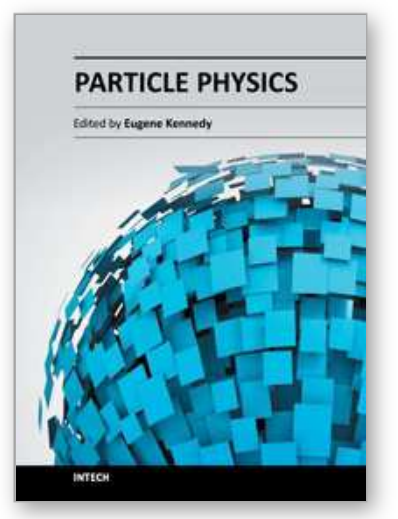

\author{
Particle Physics \\ Edited by Dr. Eugene Kennedy
}

ISBN 978-953-51-0481-0

Hard cover, 114 pages

Publisher InTech

Published online 20, April, 2012

Published in print edition April, 2012

Stimulated by the Large Hadron Collider and the search for the elusive Higgs Boson, interest in particle physics continues at a high level among scientists and the general public. This book includes theoretical aspects, with chapters outlining the generation model and a charged Higgs boson model as alternative scenarios to the Standard Model. An introduction is provided to postulated axion photon interactions and associated photon dispersion in magnetized media. The complexity of particle physics research requiring the synergistic combination of theory, hardware and computation is described in terms of the e-science paradigm. The book concludes with a chapter tackling potential radiation hazards associated with extremely weakly interacting neutrinos if produced in copious amounts with future high-energy muon-collider facilities.

\title{
How to reference
}

In order to correctly reference this scholarly work, feel free to copy and paste the following:

Brian Robson (2012). The Generation Model of Particle Physics, Particle Physics, Dr. Eugene Kennedy (Ed.), ISBN: 978-953-51-0481-0, InTech, Available from: http://www.intechopen.com/books/particle-physics/thegeneration-model-of-particle-physics

\section{INTECH}

open science | open minds

\section{InTech Europe}

University Campus STeP Ri

Slavka Krautzeka 83/A

51000 Rijeka, Croatia

Phone: +385 (51) 770447

Fax: +385 (51) 686166

www.intechopen.com

\section{InTech China}

Unit 405, Office Block, Hotel Equatorial Shanghai

No.65, Yan An Road (West), Shanghai, 200040, China 中国上海市延安西路65号上海国际贵都大饭店办公楼 405 单元

Phone: +86-21-62489820

Fax: $+86-21-62489821$ 
(C) 2012 The Author(s). Licensee IntechOpen. This is an open access article distributed under the terms of the Creative Commons Attribution 3.0 License, which permits unrestricted use, distribution, and reproduction in any medium, provided the original work is properly cited. 